\title{
OPORTUNA TOMA DE DECISIONES EN LA GESTIÓN DE CONSERVACIÓN DE LA CARRETERA
}

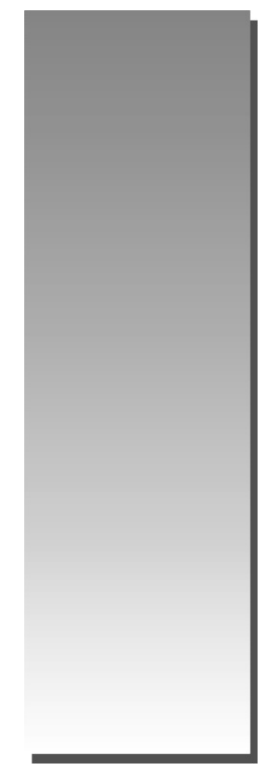

\author{
Lic. Edgar Vicente Armas* \\ evicetea@unmsm.edu.pe
}

\begin{abstract}
RESUMEN
El ciclo de una carretera está conformado por 4 fases: A) El de la construcción que puede ser sólida o con algunos defectos, una vez concluidas entran en servicio. B) El de deterioro lento y poco visible; el camino va experimentando un desgaste, principalmente en la superficie pavimentada y en menor grado, en el resto de su estructura. Esto se produce por la gran cantidad de vehículos pesados y livianos que circulan en él y por acciones propias del clima y otros factores, en esta fase la conservación vial es nula. C) Deterioro acelerado y quiebre; al inicio de esta fase, la estructura básica del camino aún sigue intacta. A medida que va avanzando esta fase, comienza a deteriorarse la estructura básica, que no es visible. Estos daños comienzan siendo puntuales, y luego se van extendiéndose hasta que finalmente afectan la mayor parte del camino, periodo entre dos y cinco años. Al inicio de esta fase se debe iniciar la conservación de la carretera cuyo costo es el $10 \%$ de la construcción original. D) Descomposición total; se caracteriza por la pérdida del pavimento, cada vez que pasa un vehículo pesado se desprenden trozos de capa asfáltica, hasta que al final termina siendo un camino de grava y a la larga, de tierra. Los vehículos comienzan a experimentar daños en los neumáticos, ejes, amortiguadores y en el chasis. En general, los costos de operación de los vehículos suben de manera considerable y la cantidad de accidentes graves también aumenta.

Existe un momento de preciso para efectuar ciertos trabajos de conservación de una carretera, en el caso de un camino con pavimento asfáltico. La toma de decisión para el refuerzo de las superficies debe efectuarse al principio de la fase C. Sin embargo, mucho más grave es si se pasa del momento oportuno para intervenir y los trabajos de conservación necesarios se postergan. El atraso, tanto en el refuerzo como en el mantenimiento rutinario, produce daños en la estructura básica del camino. En este caso, la pérdida es mucho mayor, pues el tipo de intervención necesaria será de rehabilitación, que tiene un costo mucho más alto que el correspondiente a trabajos efectuados de manera oportuna.
\end{abstract}

Palabras claves: Conservación vial (Road maintenance); Rehabilitación (Rehabilitation); Vehículos pesados (Heavy vehicles); Estructura básica (Basic structure).

\footnotetext{
* Licenciado en Administración, Economista Colegiado, Doctor en Administración, Docente de la Facultad de Ciencias Admistrativas de la
} UNMSM. 


\begin{abstract}
ABSTRAC
The cycle consists of a road four phases: A) The construction which may be solid or with some flaws, upon completion of entering service. B) The slow and impairment visible, the road is experiencing wear, mainly on the paved surface and lesser degree, the rest of their structure. This occurs because of the large number of vehicles heavy and light circulating in it and shares weather and other factors, at this stage road maintenance is nil. C) Accelerated deterioration and breakdown, at the beginning of this phase, the structure basic road still intact. As this phase progresses, begins to deteriorate the basic structure, which is not visible. These damages start being punctual, and then leave finally affect spreading until most of the way, a period between two and five years. At the start of this phase should begin conserving road whose cost is $10 \%$ of the original construction. D) Total decomposition, characterized by the loss of pavement, each After passing a heavy vehicle is off pieces of asphalt layer, until finally ends being a gravel road and eventually land. The vehicles begin to experience damage in the tire axis in the chassis and dampers. In general, the operating costs vehicles up significantly and the number of accidents increases.

There is a moment of need to perform certain maintenance work of a road, in the If a road with asphalt pavement. The decision making for reinforcing surfaces must be done at the beginning of phase $\mathrm{C}$. But much more serious is if you pass the time to intervene timely and necessary conservation work are postponed. The delay, in the reinforcement as routine maintenance, damage occurs in the basic structure of the road. In this case, the loss is much greater, because the type of intervention needed is rehabilitation, which costs much higher than that for work done in a timely manner.
\end{abstract}

Keywords: Conservation Road (Road Maintenance) Rehabilitation (Rehabilitation); Vehicles Heavy (Heavy vehicles); basic structure (basic structure).

\section{PLANTEAMIENTO}

Cuando las carreteras están en mal estado, los costos operacionales de los vehículos se incrementan en $30 \%, 50 \%$ o incluso más, lo que puede evitarse mediante una conservación vial efectiva, a un costo significativamente menor. En el largo plazo, por cada unidad monetaria no empleada oportunamente en conservación, implica tres unidades de mayores costos vehiculares y dos a tres unidades de rehabilitaciones de vías que hubieran podido evitarse. Es decir, cada dólar "ahorrado" en conservación se traduce en la pérdida de cinco o seis, que gravitan sobre el tesoro público y el sector transporte por carretera. Ello puede representar entre $1.5 \%$ y $3 \%$ del producto interno bruto anual, a lo cual se agregan otros perjuicios, como aumento de los tiempos de viaje, pérdida de mercaderías, accidentes imputables al estado de las vías, que pueden incluso duplicar dicho ya importante impacto negativo sobre la economía

\section{DESARROLLO TEMÁTICO}

Las carreteras están sometidas a un ciclo, que consta de cuatro fases:

Fase A. Construcción: Una carretera puede ser de construcción sólida, o con algunos defectos, o bien con un diseño o una ejecución de la cons- trucción claramente deficiente. De todos modos, el camino entra en servicio apenas termina la obra.

Fase B. Deterioro lento y poco visible: Durante un cierto número de años, el camino va experimentando un desgaste y un proceso de debilitamiento lento, principalmente en la superficie pavimentada, como asimismo, aunque en menor grado, en el resto de su estructura. Este desgaste se produce por la gran cantidad de vehículos pesados y livianos que circulan en él, aunque también por influencia del clima, del agua de lluvias o las aguas superficiales, la radiación solar, los cambios de la temperatura, y otros factores. Por otro lado, la velocidad del desgaste depende también de la calidad de la construcción inicial. Para frenar este proceso de desgaste y debilitamiento, es necesario aplicar, con cierta frecuencia, diferentes medidas de conservación, principalmente en el pavimento y en las obras de drenaje. Además, hay que efectuar las operaciones rutinarias de mantenimiento.

En realidad, la conservación durante este periodo es prácticamente nula, por la sencilla razón de que los recursos asignados son insuficientes, o bien por los recursos escasos existentes se destinan a la restauración de aquellos caminos que se encuentran en muy mal estado. Así, se acorta 
el período de desgaste lento y poco visible; en vez de durar 10 ó 15 años, el período dura sólo seis u ocho.

Otro factor que contribuye al abandono de las carreteras se debe al malentendido concepto del "diseño de caminos para un número determinado de años". Suele decirse que un pavimento está diseñado para 5, 10 o hasta 20 años, lo que lleva a muchas personas supongan equivocadamente que no hay que conservarlos durante ese periodo, sino sólo reconstruirlos después del tiempo estipulado.

Durante este periodo, el camino se mantiene aparentemente en buen estado y el usuario no percibe el desgaste. A pesar aumento gradual de fallas menores aisladas, el camino sigue sirviendo bien a los usuarios y está en condiciones de ser conservado, en el pleno sentido del término.

Fase C. Deterioro acelerado y quiebre: Después de varios años de uso, el pavimento y otros elementos del camino están cada vez más "agotados" y el camino entra en una etapa de deterioro acelerado y resiste cada vez menos el tránsito. Al inicio de esta fase, la estructura básica del camino aún sigue intacta, las fallas superficiales son menores, y el usuario común tiene la impresión de que el camino aún se mantiene bastante sólido, sin embargo no es así. A medida que va avanzando esta fase comienza a deteriorarse la estructura básica, que no es visible. Cuando el pavimento de un camino presenta graves fallas se puede detectar a simple vista, se puede asegurar que la estructura básica del camino también está seriamente dañada.

Estos daños comienzan siendo puntuales, y luego se van extendiéndose hasta que finalmente afectan la mayor parte del camino. La fase $C$ es relativamente corta, ya que comprende un período de entre dos y cinco años. Una vez que el daño de la superficie se generaliza, la destrucción es acelerada.

En un esquema sano de conservación, la superficie del camino debe reforzarse al inicio de la fase $\mathrm{C}$, período en el cual la condición del pavimento se torna crítica.

Normalmente basta con reforzar la superficie del camino, lo que supone un costo relativamente bajo. En los caminos pavimentados, el refuerzo suele hacerse mediante una carpeta asfáltica de entre cuatro y ocho centímetros de espesor a un costo que puede alcanzar a alrededor de $10 \%$ del valor original del camino. Sin embargo, como al comienzo de la fase $\mathrm{C}$ las fallas no son detectables a simple vista y la marcha del vehículo no es muy incómoda, generalmente no se interviene en el momento preciso, y el deterioro se agudiza.

Si dejamos pasar el momento óptimo de intervención, el simple refuerzo de la superficie ya no es suficiente. Primero deben repararse los daños que se han producido en la estructura básica del camino, lo que significa demoler y levantar las partes dañadas, remplazándolas por componentes nuevos; posteriormente se coloca el refuerzo sobre toda la superficie del camino. Cuanto más se atrase la intervención, mayores serán los daños y mayores también las reparaciones necesarias en la estructura básica del camino. Frecuentemente se utiliza el término "rehabilitación" cuando se alude a la combinación de reparaciones parciales en la estructura básica del camino con el refuerzo de su superficie.

$\mathrm{Al}$ no intervenir en momento alguno durante la fase $\mathrm{C}$, el camino llega al punto de quiebre, es decir, se produce una falla generalizada, tanto del pavimento como de la estructura básica. Durante toda la fase $\mathrm{C}$, los vehículos siguen circulando, y aunque al principio lo hacen sin ningún problema, paulatinamente los usuarios van experimentando una cantidad creciente de molestias a causa de las irregularidades de la superficie: hoyos, grietas y deformaciones. Al finalizar la fase $\mathrm{C}$ y durante la fase $D$, sólo cabe reconstruir completamente el camino, a un costo que puede equivaler a entre $50 \%$ y $80 \%$ del valor de un camino completamente nuevo.

Fase D. Descomposición total: Constituye la última etapa de su existencia y puede durar varios años. Durante ese período, lo primero que se observa es la pérdida de pavimento. Cada vez que pasa un vehículo pesado se desprenden trozos de capa asfáltica, hasta que al final termina siendo un camino de grava y a la larga, de tierra. El paso de los vehículos se dificulta, la velocidad promedio de circulación baja bruscamente y la capacidad del camino queda reducida a sólo una fracción de la original. Los vehículos comienzan a experimentar daños en los neumáticos, ejes, amortiguadores y en el chasis. En general, los costos de operación de los vehículos suben de manera considerable y la cantidad de accidentes graves también aumenta. En esta última etapa llega un momento en que los automóviles normales ya no pueden continuar transitando y sólo algunos camiones y jeeps lo 
siguen haciendo. (Anexo № 01).

Existe un momento de preciso para efectuar ciertos trabajos de conservación de una carretera. Por ejemplo, en un camino con pavimento asfáltico, el refuerzo de las superficies debe efectuarse al principio de la fase $\mathrm{C}$. Sin embargo, mucho más grave es si se pasa del momento oportuno para intervenir y los trabajos de conservación necesarios se postergan. El atraso, tanto en el refuerzo como en el mantenimiento rutinario, produce daños en la estructura básica del camino. En este caso, la pérdida es mucho mayor, pues el tipo de intervención necesaria será de rehabilitación, que tiene un costo mucho más alto que el correspondiente a trabajos efectuados de manera oportuna.

Como si lo señalado fuera poco, existe otro efecto perjudicial cuando se posterga la conservación. El deterioro de la superficie de rodadura trae como consecuencia el aumento de los costos de operación de los usuarios. Este incremento es leve cuando el camino llega a estado regular, pero aumenta rápidamente al deteriorarse el estado superficial. Todo ello se traduce en un aumento de los costos de transporte, lo que significa una presión inflacionaria de carácter estructural, $\mathrm{Si}$ se suman los sobrecostos que afectan a todos los vehículos que circulan por una red vial degradada, se suele comprobar que esa cifra supera el $1 \% \mathrm{del}$ producto bruto del país. En otras palabras, en ese caso se derrocha una enorme cantidad de recursos que podrían tener mejor destino.

En consecuencia y al contrario de lo que se puede pensar a simple vista, aplicar una política de conservación sana representa, especialmente para los países en desarrollo, ahorrar ingentes recursos, que serian útiles para su mejor desenvolvimiento.

Es evidente entonces la necesidad de identificar el momento el preciso. para cada trabajo de conservación, especialmente cuando se trata de caminos pavimentados. En el caso de los caminos de tierra o de ripio, por sus características técnicas, puede contarse con un margen de tiempo más flexible para efectuar las intervenciones.

Establecer el momento oportuno en que debe realizarse cada medida de conservación no es de ninguna manera algo trivial o sencillo, considerando que están en juego cifras significativas para la economía. De hecho se requiere emplear complejos instrumentos de análisis para computar los costos de conservación y de operación que podrían generarse al adoptar diferentes opciones y determinar cuál es, para cada camino, el estado o condición límite que a largo plazo reduce al mínimo la suma total de los costos de los usuarios y de la agencia vial. Por ello es paradojal que la conservación, actividad de gran complejidad y relevancia para un país, haya tenido poco prestigio, ya que tradicionalmente ha sido la "pariente pobre" del quehacer vial. (Anexo № 02 y 03).

\section{CONCLUSIONES}

1. La vida útil de una carretera está determinado por cuatro fases: A) Construcción, B) Deterioro Lento y Poco Visible, C) Deterioro Acelerado y Quiebre y D) Destrucción Total. (Ver gráfico № 01) Caso carretera pavimentada.

2. La conservación vial es una actividad cuya finalidad es mantener la transitabilidad de las carreteras a fin de facilitar el tránsito vehicular y los servicios de transporte sean oportunos y eficientes.

3. Existe un momento oportuno para tomar decisiones para realizar el mantenimiento de las vías y esta es al inicio de la Fase $C$, el costo de esta intervención representa el $10 \%$ del costo de construcción de la carretera. Postergar dicha actividad trae como consecuencia incremento en los costos de la operación vehicular, demora en los tiempos de viaje y pérdida para el estado.

4. El costo de restablecer la transitabilidad de la carretera cuando no es en su momento oportuno representa el $50 \%$ a $80 \%$ del costo de la construcción original de la carretera.

5. La conservación vial en la Fase $\mathrm{C}$ es prácticamente nula, por la sencilla razón de que los recursos asignados son insuficientes, o bien por los recursos escasos existentes se destina a la restauración de aquellos caminos que se encuentran en muy mal estado. Así, se acorta el período de desgaste lento y poco visible; en vez de durar 10 ó 15 años, el período dura sólo seis u ocho.

6. En nuestro país por las características geográficas y la variedad de climas la conservación de las carreteras es muy importante, sin embargo estas no son prioritarios por las autoridades de turno, por lo que no tiene réditos políticos, dado que la conservación vial no se inaugura, en tanto que sí la construcción, rehabilitación y mejoramiento de carreteras. 
7. Se debe crear una cultura de la conservación vial y en esto es muy importante la labor de las Universidades de los Centros de Investigación ya que una carretera bien conservada, garantiza crecimiento y desarrollo de la economía, Nacional, Regional y Local.

\section{LITERATURA CITADA}

CEPAL-GTZ CAMINOS : Gestión y Conservación de Redes Viales año 1994
BULL ALBERTO Reforma de Conservación VialNuevo Concepto sobre Gestión de Conservación Vial Boletin № 01 Julio de 1995.

BULL ALBERTO Reforma de Conservación VialConservación Vial Financiado por los Usuarios; Boletin № 04 Enero de 1997.

ZIETLOW GUNTER Reforma de Conservación Vial Boletin № 01 Julio de 1995. 


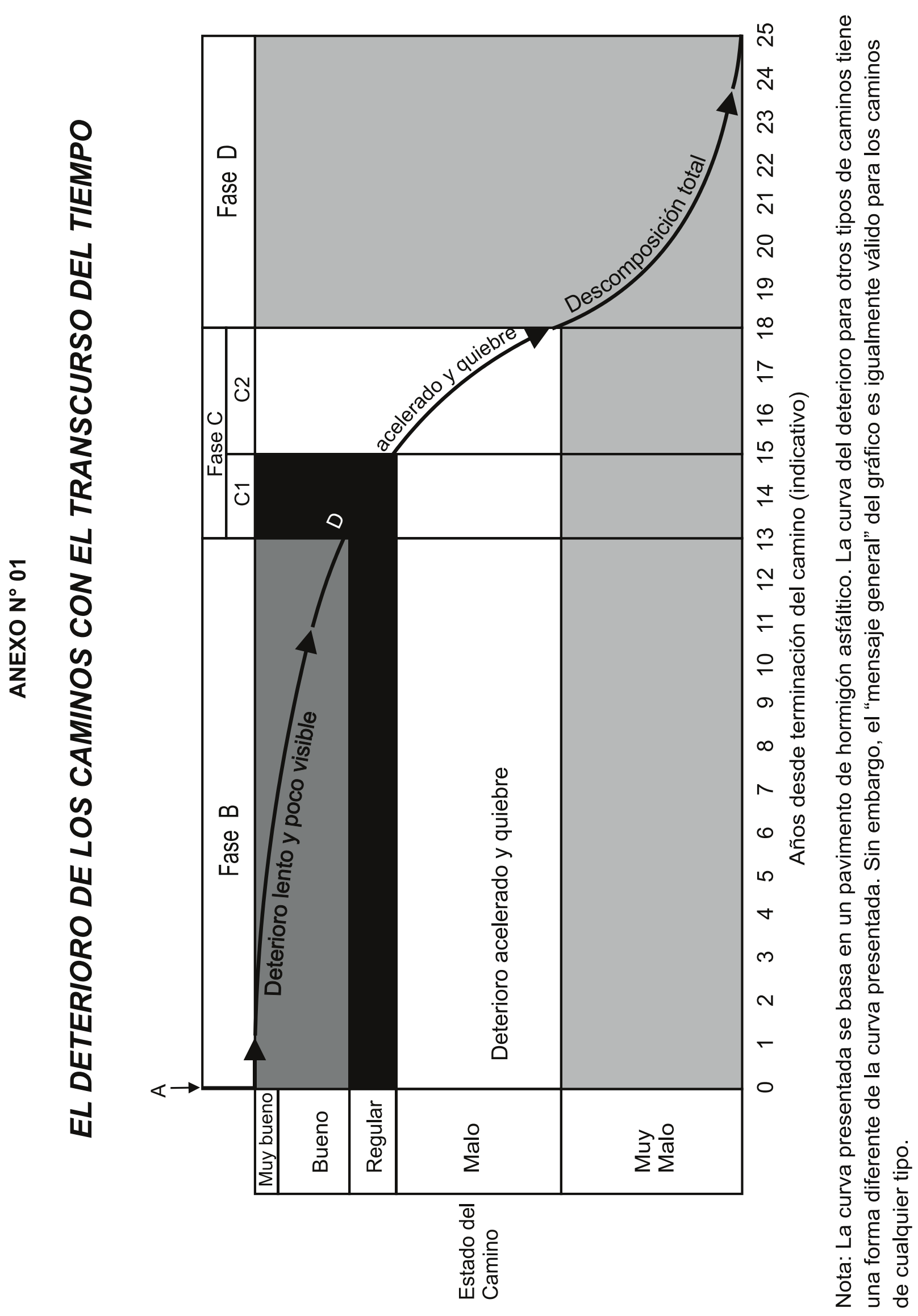




\section{A: EFECTOS PARA LA AGENCIA VIAL}

(Gastos en caminos)

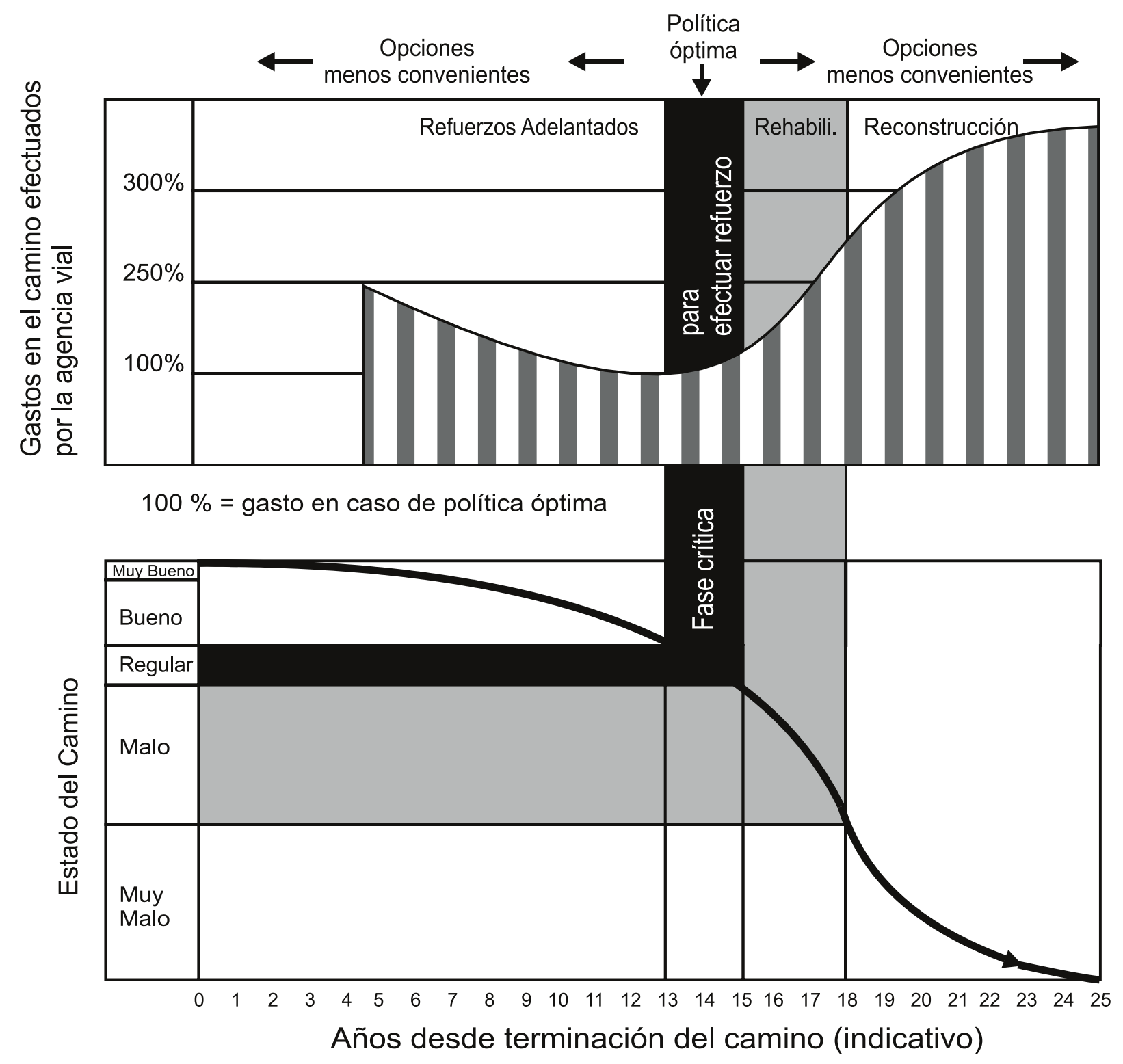




\section{B: EFECTOS PARA LOS USUARIOS}

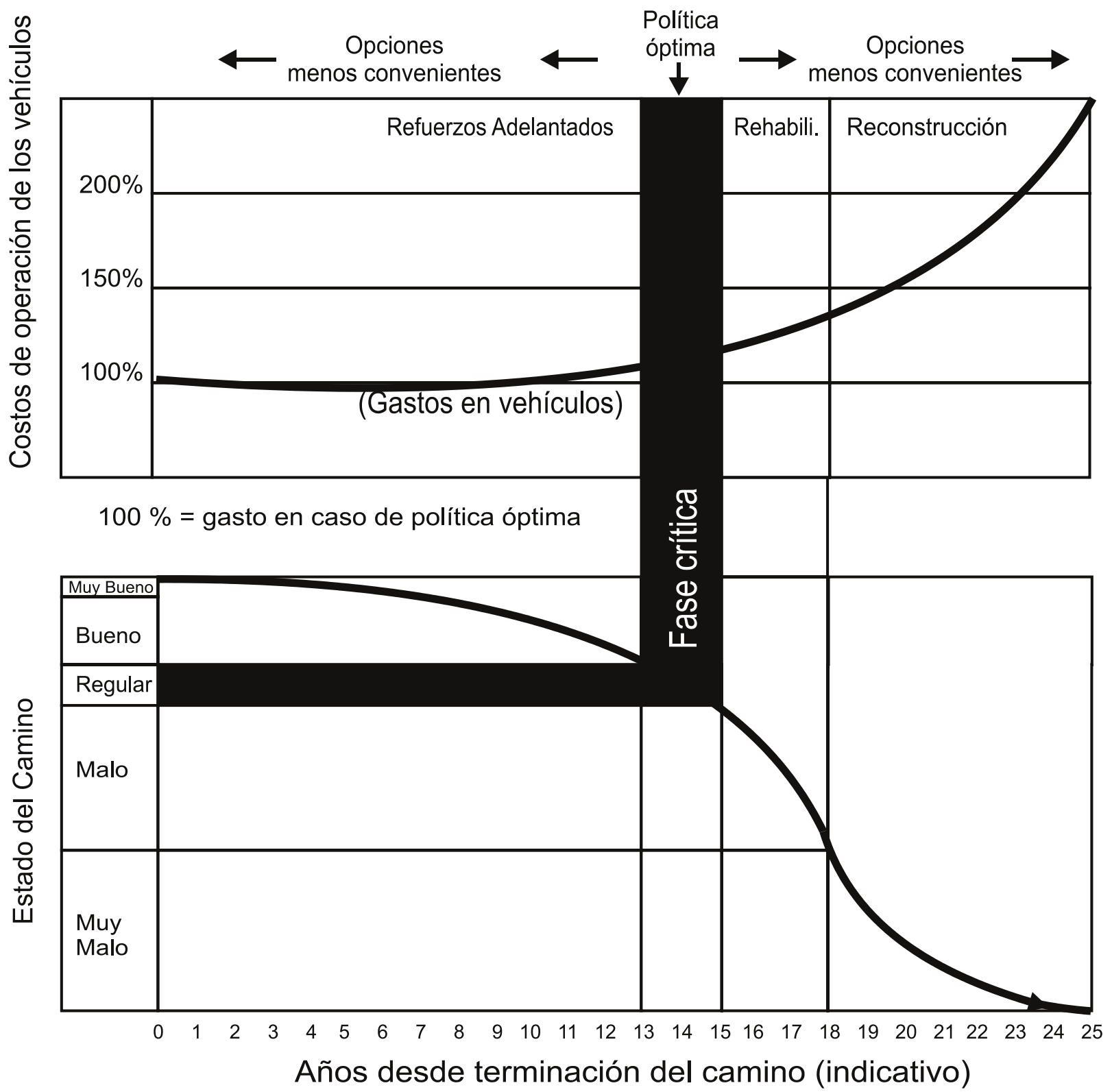

